\title{
Stability Analysis of a Mathematical Model for the Use of Wolbachia to Stop the Spread of Zika Virus Disease
}

\author{
Michael C. Anyanwu ${ }^{1}$, Godwin C. Mbah ${ }^{2}$ \\ ${ }^{1}$ Department of Mathematics, Michael Okpara University of Agriculture, Umudike, \\ Abia State, Nigeria, e-mail: manyanwu71@yahoo.com \\ ${ }^{2}$ Department of Mathematics, University of Nigeria, Nsukka, Enugu State, Nigeria, \\ e-mail: godwin.mbah@unn.edu.ng
}

\section{SUMMARY}

The use of wolbachia-infected mosquitoes to stop the spread of zika virus disease is modeled and analyzed. The model consists of a system of 10 ordinary differential equations which describes the dynamics of the disease in the human population, a wolbachia-free Aedes aegypti population, and a wolbachia-infected Aedes aegypti population used for disease control. A stability analysis of the disease-free equilibrium is conducted, which shows that it is both locally and globally asymptotically stable when the reproduction number is less than one. The result of the stability analysis shows that the spread of zika virus disease can be stopped, irrespective of the initial sizes of the infected human and mosquito populations, when wolbachia-infected Aedes aegypti are introduced in the area where the disease is endemic.

Key words: zika virus disease, Aedes aegypti, wolbachia, global stability

\section{Introduction}

Zika virus disease is one of the flavivirus diseases transmitted to humans mainly through the bites of infected female Aedes aegypti mosquitoes (CaoLormeau et al., 2014). The virus can equally be transmitted from human to human through sex, blood transfusion and mother-to-fetus transmission (Musso et al., 2014). The disease has a negligible mortality rate; hence, it was not considered a serious disease until its linking with an increase in microcephaly in babies born to mothers who were infected with the virus during pregnancy (Mlakar et al., 2016; Cauchmez et al., 2016). In a recent outbreak of zika in Brazil, there were over 5000 confirmed cases of microcephaly (Gao et al., 2016). In addition to microcephaly, zika has been 
linked to the neurological disorder Guillain-Barre Syndrome (GBS) (CaoLormeau et al., 2016). These health conditions associated with zika virus disease prompted the World Health Organization (WHO) to declare the disease a Public Health Emergency of International Concern in February 2015 (WHO, 2016). At present, there is no known cure for zika virus disease, and the idea of using bed nets to reduce mosquito bites cannot be applied in this case, because Aedes aegypti mosquitoes are day-biters. Also, physical and chemical control methods have not proved to be effective in controlling other mosquito-borne diseases such as malaria and dengue. Hence, it seems that the only feasible option is to adopt biological control by introducing wolbachia-infected Aedes aegypti mosquitoes in the area where zika virus disease is endemic.

Wolbachia is a group of bacteria naturally found in the reproductive tissues of some arthropods. They are transmitted maternally through the cytoplasm of eggs of their hosts. Some strains of wolbachia such as wMel reduce the ability of the host insects to transmit disease-causing pathogens to humans. This is the idea behind using wolbachia-infected mosquitoes as a bio-control for zika virus disease. Wolbachia are not found naturally in the Aedes aegypti mosquitoes that transmit zika virus. In order to use this method to control the spread of zika virus disease, Aedes aegypti mosquitoes have to be manually infected with wolbachia in the laboratory (Hughes et al., 2013) and then released into the wild to mate with wolbachia-free insects. When wolbachia is present in infected mosquitoes, it increases the incubation period (or reduces the incubation rate) of the virus, thereby increasing the time it takes the mosquito carrying the virus to become infectious. Since the adult life of the mosquito is short, most of the mosquitoes carrying the virus die before they become infectious. Therefore, the infected wolbachia-carrier mosquitoes may not transmit the virus to humans through bites before they die. In addition, wolbachia induces cytoplasmic incompatibility (CI), which helps its host to invade the wolbachia-free Aedes aegypti mosquito population in the wild (Koiller et al., 2014). CI is a phenomenon which prevents the development of embryos (causes non-hatching of eggs) when wolbachia-carrier male mosquitoes mate with wolbachia-free female mosquitoes or with female mosquitoes carrying a different wolbachia strain (Mains et al., 2013). The resultant effect of cytoplasmic incompatibility on the mosquitoes' reproduction process is that wolbachia-carrier female mosquitoes will have the advantage of producing many more offspring than the wild insects, since they can mate with all males in the population 
whether infected or not infected with wolbachia, to produce viable eggs that are infected with wolbachia (Hancock et al., 2011). In this work, we present a model for the transmission dynamics of zika virus and its control with wolbachia-carrier Aedes aegypti mosquitoes. Essentially, we show that the disease-free equilibrium, that is the state when there is no disease in the populations, is both locally and globally stable when the control reproduction number is less than one. This is essential to ascertain the efficacy of using this method to stop the spread of zika virus disease.

The rest of this work is organized as follows: The model with its basic assumptions is presented in section 2. In section 3, we analyze the model by determining the disease-free and endemic equilibrium points, obtaining the expression for the control reproduction number, and carry out local and global asymptotic stability analyses of the disease-free state. In section 4, some graphical representations of the dynamics of zika virus disease are shown. Section 5 concludes the work.

\section{Model assumptions and formulation}

In this model, we assume that humans can only contract zika virus when bitten by infectious female Aedes aegypti mosquitoes. This means that other modes of transmission of the virus to humans, such as unsafe sex, unsafe blood transfusion or perinatal transmission, are not included in the model. On the other hand, transmission of the zika virus from human to mosquito occurs when an adult, uninfected female Aedes aegypti mosquito bites a human to suck blood. If the human is already infected with the virus, it may be passed to the mosquito. The mosquito, once infected, remains so and continues to infect humans throughout its lifetime. Therefore, the model includes three major populations: the human population, the adult female wolbachia-free Aedes aegypti population, and the adult female wolbachiacarrier Aedes aegypti population used for disease control.

\subsection{Zika dynamics in human population}

The total human population, $N_{H}(t)$, at any time $t$, is divided into 4 compartments or classes, namely, (i) the susceptible class, $S_{H}(t)$, (ii) the latent or the exposed class, $E_{H}(t)$, (iii) the infectious class, $I_{H}(t)$, and (iv) the recovered class, $R_{H}(t)$. We assume that individuals in the human population are recruited into the susceptible class through migration into the zika endemic area at the rate $\Pi_{H}$. The susceptible class acquires zika virus through 
infectious wolbachia-free mosquito bites at the rate $b_{1}$, with transmission probability $\alpha_{M H}$. Susceptible humans may also contract the virus through the bites of wolbachia-carrier mosquitoes at the rate $b_{2}$, with a very negligible probability of infection, $\alpha_{M w H} \ll \alpha_{M H}$. After the incubation period has elapsed, the exposed class becomes infectious at the incubation rate $\beta_{H}$, but recovers at the rate $\gamma$. All of the classes in the human population are subject to the natural mortality rate $\sigma_{H}$, whereas only the infectious class is affected by additional death induced by the disease, at the rate $\sigma_{H}^{\prime}$, which is negligible.

\subsection{Zika dynamics in the Aedes aegypti population}

The population of wolbachia-free adult female Aedes aegypti mosquitoes is grouped into three classes, namely (i) susceptible mosquitoes, $S_{M}(t)$, (ii) exposed mosquitoes, $E_{M}(t)$ and (iii) infectious mosquitoes, $I_{M}(t)$. The wolbachia-free male mosquitoes mate with their female counterparts in the wolbachia-free and wolbachia-carrier mosquito populations to produce wolbachia-free and wolbachia-carrier offspring, respectively. The female wolbachia-free mosquitoes join the susceptible class through migration at the rate $\Pi_{M}$, or through oviposition at the rate $\mu_{M}$. To model the effect of cytoplasmic incompatibility, we assume that the proportion $q$ of the eggs produced by the female wolbachia-free mosquitoes are viable, while $(1-q)$ are non-viable. This parameter is responsible for the reduction in the population of the wild mosquitoes. Susceptible mosquitoes contract zika virus when they bite humans in the infectious classes at the biting rate $b_{1}$, with probability of infection $\alpha_{H M}$, and move to the exposed class. After the incubation period, the exposed mosquitoes become infectious and move to the infectious class at the incubation rate $\beta_{M}$. The mosquitoes remain infectious throughout their lifetime until they die naturally at the rate $\sigma_{M}$. Similarly, the female adult wolbachia-carrier Aedes aegypti mosquitoes are grouped in the same manner, with the following compartments: the susceptible wolbachia-carrier class, $S_{M w}(t)$; the exposed wolbachia-carrier class, $E_{M w}(t)$; and the infectious wolbachia-carrier class, $I_{M w}(t)$. The dynamics of zika virus disease in the wolbachia-carrier mosquito population is similar to that in the wolbachia-free mosquitoes, except at the infectious stage, where the probability of the wolbachia-carrier mosquitoes transmitting the virus to susceptible humans is negligible. We also note that susceptible wolbachia-infected mosquitoes are introduced from the laboratory into the zika-endemic area at the constant rate $\Pi_{M w}$. Using the above assumptions 
on the transmission dynamics and control of zika, we obtain the following system of nonlinear ordinary differential equations which model the dynamics of the disease.

$$
\begin{aligned}
\frac{d S_{H}(t)}{d t} & =\Pi_{H}-b_{1} \alpha_{M H} S_{H}(t) I_{M}(t)-b_{2} \alpha_{M w H} I_{M w}(t) S_{H}(t)-\sigma_{H} S_{H}(t), \\
\frac{d E_{H}(t)}{d t} & =b_{1} \alpha_{M H} I_{M}(t) S_{H}(t)+b_{2} \alpha_{M w H} I_{M w}(t) S_{H}(t)-\left(\beta_{H}+\sigma_{H}\right) E_{H}(t), \\
\frac{d I_{H}(t)}{d t} & =\beta_{H} E_{H}(t)-\left(\gamma+\sigma_{H}+\sigma_{H}^{\prime}\right) I_{H}(t), \\
\frac{d R_{H}(t)}{d t} & =\gamma I_{H}(t)-\sigma_{H} R_{H}(t), \\
\frac{d S_{M}(t)}{d t} & =\Pi_{M}+q \kappa \mu_{M} N_{M}(t)-b_{1} \alpha_{H M} S_{M}(t) I_{H}-\sigma_{M} S_{M}(t), \\
\frac{d E_{M}(t)}{d t} & =b_{1} \alpha_{H M} S_{M}(t) I_{H}(t)-\left(\beta_{M}+\sigma_{M}\right) E_{M}(t), \\
\frac{d I_{M}(t)}{d t} & =\beta_{M} E_{M}(t)-\sigma_{M} I_{M}(t), \\
\frac{d S_{M w}(t)}{d t} & =\Pi_{M w}+\kappa \mu_{M w} N_{M w}(t)-b_{2} \alpha_{H M w} S_{M w}(t) I_{H}(t)-\sigma_{M} S_{M w}(t), \\
\frac{d E_{M w}(t)}{d t} & =b_{2} \alpha_{H M w} S_{M w}(t) I_{H}(t)-\left(\beta_{M w}+\sigma_{M}\right) E_{M w}(t), \\
\frac{d I_{M w}(t)}{d t} & =\beta_{M w} E_{M w}(t)-\sigma_{M} I_{M w}(t)
\end{aligned}
$$

The initial conditions are $S_{H}(0)=S_{H}^{0}, E_{H}(0)=E_{H}^{0}, I_{H}(0)=I_{H}^{0}, R_{H}(0)=$ $R_{H}^{0}, S_{M}(0)=S_{M}^{0}, E_{M}(0)=E_{M}^{0}, I_{M}(0)=I_{M}^{0}, S_{M w}(0)=S_{M w}^{0}, E_{M w}(0)=$ $E_{M w}^{0}, I_{M w}(0)=I_{M w}^{0}$, which we assume to be nonnegative quantities. The domain of existence of a solution to this system can be described as $\mathcal{D}=$ $D_{1} \cup D_{2} \cup D_{3}$, where $D_{1}=\left\{S_{H}, E_{H}, I_{H}, R_{H}\right) \in \mathbb{R}_{+}^{4} \mid S_{H}+E_{H}+I_{H}+$ $\left.R_{H} \leqslant N_{H}\right\}, D_{2}=\left\{\left(S_{M}, E_{M}, I_{M}\right) \in \mathbb{R}_{+}^{3} \mid S_{M}+E_{M}+I_{M} \leqslant N_{M}\right\}$, and $D_{3}=$ $\left\{\left(S_{M w}, E_{M w}, I_{M w}\right) \in \mathbb{R}_{+}^{3} \mid S_{M w}+E_{M w}+I_{M w} \leqslant N_{M w}\right\}$.

From (1), we see that the total populations of humans and of wolbachia-free and wolbachia-carrier mosquitoes, respectively, satisfy the equations

$$
\begin{aligned}
\frac{d N_{H}}{d t} & =\Pi_{H}-\sigma_{H} N_{H}-\sigma_{H}^{\prime} I_{H}, \\
\frac{d N_{M}}{d t} & =\Pi_{M}+\left(q \kappa \mu_{M}-\sigma_{M}\right) N_{M}, \\
\frac{d N_{M w}}{d t} & =\Pi_{M w}+\left(\kappa \mu_{M w}-\sigma_{M}\right) N_{M w} .
\end{aligned}
$$


Let $f(X(t))$ represent the right-hand side of (1), where

$X(t)=\left(S_{H}, E_{M}, I_{H}, R_{H}, S_{M}, E_{M}, I_{M}, S_{M w}, E_{M w}, I_{M w}\right)$. It is easy to show that $f(X(t))$ is continuous, and has continuous partial derivatives with respect to each of the state variables. Hence, there exists a unique solution $X(t)$ to the system (1) that passes through the initial solution, $X_{0}$.

\subsection{Positivity of solution}

Since the model describes the dynamics of zika virus disease in human and mosquito populations, it will be epidemiologically and mathematically meaningful if all of the state variables (all human and mosquito subpopulations) are nonnegative $\forall t \geqslant 0$. Moreover, given that the initial conditions are nonnegative in the octant $\mathcal{D}$, we need to establish that the solution remains positive in the set. From the first equation in (1), we have

$$
\begin{aligned}
& \frac{d S_{H}(t)}{d t}>-b_{1} \alpha_{M H} S_{H} I_{M}-b_{2} \alpha_{M w H} I_{M w} S_{H}-\sigma_{H} S_{H} \\
\Longrightarrow & \frac{d S_{H}(t)}{S_{H}(t)}>-\left(b_{1} \alpha_{M H} I_{M}+b_{2} \alpha_{M w H} I_{M w}+\sigma_{H}\right) d t \\
\Longrightarrow & S_{H}(t)>S_{H}^{0} e^{-\int^{t}\left(b_{1} \alpha_{M H} I_{M}(s)+b_{2} \alpha_{M w H(s)} I_{M w(s)}+\sigma_{H}\right) d s}>0
\end{aligned}
$$

Also from the second equation, we have that

$$
\begin{aligned}
& \frac{d E_{H}(t)}{d t}>-\left(\beta_{H}+\sigma_{H}\right) E_{H} \Longrightarrow \frac{d E_{H}(t)}{E_{H}(t)}>-\left(\beta_{H}+\sigma_{H}\right) d t \\
& \Longrightarrow \quad E_{H}(t)>E_{H}^{0} e^{-\left(\beta_{H}+\sigma_{H}\right) t}>0
\end{aligned}
$$

A similar argument can be used to show that other component solutions are positive. Therefore, the solution remains positive in $\mathcal{D} \forall t>0$.

\subsection{Positive invariant region}

To show that $\mathcal{D}$ is positive invariant with respect to the flow of the model system, we use the total populations in (2). It is easy to show from (2) that

$$
\begin{aligned}
N_{H}(t) & \leqslant \frac{\Pi_{H}}{\sigma_{H}}-\left(\frac{\Pi_{H}}{\sigma_{H}}-N_{H}^{0}\right) e^{-\left(\sigma_{H}-\mu_{H}\right) t} \\
N_{M}(t) & \leqslant \frac{\Pi_{M}}{\sigma_{M}-\mu_{M}}-\left(N_{M}^{0}-\frac{\Pi_{M}}{\sigma_{M}-\mu_{M}}\right) e^{-\left(\sigma_{M}-q k \mu_{M}\right) t} \\
N_{M w}(t) & \leqslant \frac{\Pi_{M w}}{\sigma_{M}-\mu_{M w}}-\left(N_{M w}^{0}-\frac{\Pi_{M w}}{\sigma_{M}-\mu_{M w}}\right) e^{-\left(\sigma_{M}-k \mu_{M w}\right) t}
\end{aligned}
$$


Hence, we have that

$$
\begin{aligned}
\lim _{t \rightarrow \infty} \sup N_{H}(t) & \leqslant \frac{\Pi_{H}}{\sigma_{H}} \\
\lim _{t \rightarrow \infty} \sup N_{M}(t) & \leqslant \frac{\Pi_{M}}{\sigma_{M}-q k \mu_{M}} \\
\lim _{t \rightarrow \infty} \sup N_{M w}(t) & \leqslant \frac{\Pi_{M w}}{\sigma_{M}-k \mu_{M w}}
\end{aligned}
$$

Since the total human and mosquito populations are bounded above, and no population is negative, this implies that all solutions enter and remain in the domain $\mathcal{D} \quad \forall t \geqslant 0$. This shows that $\mathcal{D}$ is positive invariant. The positivity of other solutions can be shown in a similar manner. Therefore, the solution remains positive $\forall t>0$.

\section{Analysis of the model}

\subsection{Disease-free equilibrium and the control reproduction number}

The disease-free equilibrium, $E_{0}$ of the model is the steady state of (1) in the absence of zika virus disease in the population. Hence, it is easy to see that $E_{0}=\left(\frac{\Pi_{H}}{\sigma_{H}}, 0,0,0, \frac{\Pi_{M}}{\sigma_{M}-q k \mu_{M}}, 0,0, \frac{\Pi_{M w}}{\sigma_{M}-k \mu_{M w}}, 0,0\right)$. Also, at disease-free equilibrium, $\left(N_{H}^{0}, N_{M}^{0}, N_{M w}^{0}\right)=\left(\frac{\Pi_{H}}{\sigma_{H}}, \frac{\Pi_{M}}{\sigma_{M}-q k \mu_{M}}, \frac{\Pi_{M w}}{\sigma_{M}-k \mu_{M w}}\right)$. The control reproduction number of a disease outbreak is the average number of persons that can be infected by a single index case when introduced in a purely susceptible population in the presence of control measures. A control measure is effective if the control reproduction number is less than one, otherwise it is ineffective. To determine $\mathcal{R}_{c}$, we shall adopt the next generation matrix approach introduced by Diekmann et al. (2010), where the reproduction number is found to be the spectral radius of a next generation matrix. If we write (1) in the form

$$
X_{i}^{\prime}(t)=\mathcal{F}_{i}(x)-\mathcal{V}_{i}(x)
$$

where $\mathcal{F}_{i}(x)$ are the terms for the rate of appearance of new infections in compartment $i$, while $\mathcal{V}_{i}(x)$ are the terms for the rate of transfer of individuals into and out of compartment $i$ by any other means including death, then the next generation matrix is given by $\mathcal{K}=F V^{-1}$, where $F=\left.\frac{\partial \mathcal{F}(x)}{\partial x}\right|_{E_{0}}$ and $V=\left.\frac{\partial \mathcal{V}(x)}{\partial x}\right|_{E_{0}}$ and the control reproduction number is therefore the spectral radius of $F V^{-1}: \mathcal{R}_{c}=\rho\left(F V^{-1}\right)$. 
Theorem 1. The next generation matrix of the model is of the form

$$
\mathcal{K}=\left(\begin{array}{ccc}
0 & \mathcal{K}_{M H} & \mathcal{K}_{M w H} \\
\mathcal{K}_{H M} & 0 & 0 \\
\mathcal{K}_{H M w} & 0 & 0
\end{array}\right)
$$

and the control reproduction number is

$$
\mathcal{R}_{C}=\sqrt{\mathcal{K}_{M H} \mathcal{K}_{H M}+\mathcal{K}_{M w H} \mathcal{K}_{H M w}}
$$

where: $\mathcal{K}_{H M}=$ the number of wolbachia-free mosquitoes that one infectious human can infect throughout his infectious lifetime, when introduced in a purely susceptible wolbachia-free mosquito population,

$\mathcal{K}_{H M w}=$ the number of wolbachia-carrier mosquitoes that one infectious human can infect throughout his infectious lifetime, when introduced in a purely susceptible wolbachia-carrier mosquito population,

$\mathcal{K}_{M H}=$ the number of humans that one infectious wolbachia-free mosquito can infect throughout its infectious lifetime, when introduced in a purely susceptible human population,

$\mathcal{K}_{M w H}=$ the number of humans that one infectious wolbachia-carrier mosquito can infect throughout its infectious lifetime, when introduced in a purely susceptible human population, with

$$
\begin{aligned}
& \mathcal{K}_{H M}=\frac{\beta_{H} b_{1} \alpha_{H M} \Pi_{M}}{\left(\sigma_{M}-q k \mu_{M}\right)\left(\beta_{H}+\sigma_{H}\right)\left(\gamma+\sigma_{H}+\sigma_{H}^{\prime}\right)}, \\
& \mathcal{K}_{H M w}=\frac{\beta_{H} b_{2} \alpha_{H M w} \Pi_{M w}}{\left(\sigma_{M}-k \mu_{M w}\right)\left(\beta_{H}+\sigma_{H}\right)\left(\gamma+\sigma_{H}+\sigma_{H}^{\prime}\right)}, \\
& \mathcal{K}_{M H}=\frac{\beta_{M} b_{1} \alpha_{M H} \Pi_{H}}{\sigma_{H} \sigma_{M}\left(\beta_{M}+\sigma_{M}\right)}, \mathcal{K}_{M w H}=\frac{\beta_{M w} b_{2} \alpha_{M w H} \Pi_{H}}{\sigma_{H} \sigma_{M}\left(\beta_{M w}+\sigma_{M}\right)} .
\end{aligned}
$$

Proof. The shape of the next generation matrix (4) is informed by the major assumptions made in the model. It is assumed that humans can transmit zika virus to wolbachia-free and wolbachia-carrier mosquitoes, and vice versa. Moreover, we have that

$$
\mathcal{F}_{i}=\left(\begin{array}{c}
b_{1} \alpha_{M H} I_{M} S_{H}+b_{2} \alpha_{M w H} I_{M w} S_{H} \\
0 \\
b_{1} \alpha_{H M} S_{M}(t) I_{H} \\
0 \\
b_{2} \alpha_{H M w} S_{M w}(t) I_{H} \\
0
\end{array}\right) \text { and } \mathcal{V}_{i}=\left(\begin{array}{c}
\left(\beta_{H}+\sigma_{H}\right) E_{H}, \\
-\beta_{H} E_{H}+\left(\gamma+\sigma_{H}+\sigma_{H}^{\prime}\right) I_{H}, \\
\left(\beta_{M}+\sigma_{M}\right) E_{M}(t), \\
-\beta_{M} E_{M}(t)+\sigma_{M} I_{M}(t), \\
\left(\beta_{M w}+\sigma_{M}\right) E_{M w}(t), \\
-\beta_{M w} E_{M w}(t)+\sigma_{M} I_{M w}(t)
\end{array}\right)
$$


from which we obtain

$$
\begin{aligned}
& F=\left(\begin{array}{cccccc}
0 & 0 & 0 & b_{1} \alpha_{M H} S_{H}^{0} & 0 & b_{2} \alpha_{M w H} S_{H}^{0} \\
0 & 0 & 0 & 0 & 0 & 0 \\
0 & b_{1} \alpha_{H M} S_{M}^{0} & 0 & 0 & 0 & 0 \\
0 & 0 & 0 & 0 & 0 & 0 \\
0 & b_{2} \alpha_{H M w} S_{M w}^{0} & 0 & 0 & 0 & 0 \\
0 & 0 & 0 & 0 & 0 & 0
\end{array}\right) \\
& V=\left(\begin{array}{cccccc}
\beta_{H}+\sigma_{H} & 0 & 0 & 0 & 0 & 0 \\
-\beta_{H} & \gamma+\sigma_{H}+\sigma_{H}^{\prime} & 0 & 0 & 0 & 0 \\
0 & 0 & \beta_{M}+\sigma_{M} & 0 & 0 & 0 \\
0 & 0 & -\beta_{M} & \sigma_{M} & 0 & 0 \\
0 & 0 & 0 & 0 & \beta_{M w}+\sigma_{M} & 0 \\
0 & 0 & 0 & 0 & -\beta_{M w} & \sigma_{M}
\end{array}\right)
\end{aligned}
$$

Then, with $F$ and $V$, the next generation matrix is

$$
\begin{aligned}
& \mathcal{K}=
\end{aligned}
$$

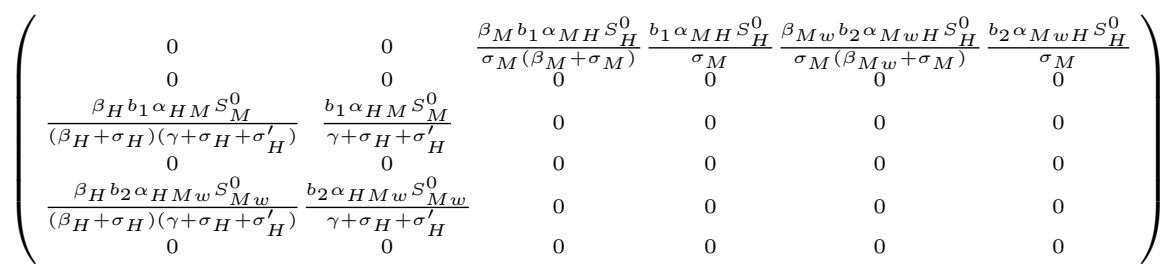

We can recover (4) by pre- and post-multiplying the next generation matrix

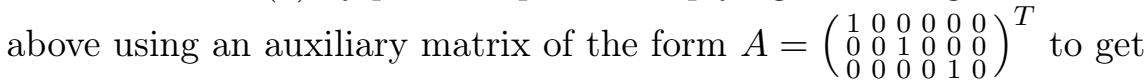

$$
\mathcal{K}_{s}=A \mathcal{K} A^{T}=\left(\begin{array}{ccc}
0 & \frac{\beta_{M} b_{1} \alpha_{M H} S_{H}^{0}}{\sigma_{M}\left(\beta_{M}+\sigma_{M}\right)} & \frac{\beta_{M w} b_{2} \alpha_{M w H} S_{H}^{0}}{\sigma_{M}\left(\beta_{M w}+\sigma_{M}\right)} \\
\frac{\beta_{H} b_{1} \alpha_{H M} S_{M}^{0}}{\left(\beta_{H}+\sigma_{H}\right)\left(\gamma+\sigma_{H}+\sigma_{H}^{\prime}\right)} & 0 & 0 \\
\frac{\beta_{H} b_{2} \alpha_{H} S_{M w} S_{M w}^{0}}{\left(\beta_{H}+\sigma_{H}\right)\left(\gamma+\sigma_{H}+\sigma_{H}^{\prime}\right)} & 0 & 0
\end{array}\right) .
$$

We see that (5) is the spectral radius of (6) or the control reproduction number of the disease. 


\subsection{Local asymptotic stability of the disease-free equilibrium}

The linearization of the model system (1) around the equilibrium point $E_{0}$ gives the Jacobian matrix:

$$
J\left(E_{0}\right)=\left(\begin{array}{cccccccccc}
-J_{11} & 0 & 0 & 0 & 0 & 0 & -J_{12} & 0 & 0 & -J_{13} \\
0 & -J_{21} & 0 & 0 & 0 & 0 & J_{22} & 0 & 0 & J_{23} \\
0 & J_{31} & -J_{32} & 0 & 0 & 0 & 0 & 0 & 0 & 0 \\
0 & 0 & J_{41} & -J_{42} & 0 & 0 & 0 & 0 & 0 & 0 \\
0 & 0 & -J_{51} & 0 & -J_{52} & 0 & 0 & 0 & 0 & 0 \\
0 & 0 & J_{61} & 0 & 0 & -J_{62} & 0 & 0 & 0 & 0 \\
0 & 0 & 0 & 0 & 0 & J_{71} & -J_{72} & 0 & 0 & 0 \\
0 & 0 & -J_{81} & 0 & 0 & 0 & 0 & -J_{82} & 0 & 0 \\
0 & 0 & J_{91} & 0 & 0 & 0 & 0 & 0 & -J_{92} & 0 \\
0 & 0 & 0 & 0 & 0 & 0 & 0 & 0 & J_{101} & -J_{102}
\end{array}\right)
$$

where:

$J_{11}=\sigma_{H}, J_{12}=b_{1} \alpha_{M H} S_{H}^{0}, J_{13}=b_{2} \alpha_{M w H} S_{H}^{0}, J_{21}=\left(\beta_{H}+\sigma_{H}\right), J_{22}=$ $b_{1} \alpha_{M H} S_{H}^{0}, \quad J_{23}=b_{2} \alpha_{M w H} S_{H}^{0}, \quad J_{31}=\beta_{H}, J_{32}=\left(\gamma+\sigma_{H}+\sigma_{H}^{\prime}\right), J_{41}=$ $\gamma, J_{42}=\sigma_{H}, J_{51}=b_{1} \alpha_{H M} S_{M}^{0}, J_{52}=\left(\sigma_{M}-q k \mu_{M}\right), J_{61}=b_{1} \alpha_{H M} S_{M}^{0}, J_{62}=$ $\left(\beta_{M}+\sigma_{M}\right), J_{71}=\beta_{M}, J_{72}=\sigma_{M}, J_{81}=b_{2} \alpha_{H M w} S_{M w}^{0}, J_{82}=\left(\sigma_{M}-\right.$ $\left.k \mu_{M w}\right), \quad J_{91}=b_{2} \alpha_{H M w} S_{M w}^{0}, J_{92}=\left(\beta_{M w}+\sigma_{M}\right), J_{101}=\beta_{M w}, J_{102}=\sigma_{M}$. The eigenvalues of $J\left(E_{0}\right)$ are $-J_{11}<0,-J_{42}<0,-J_{52}<0,-J_{82}<0$ and the eigenvalues of the $6 \times 6$ submatrix $J^{\prime}\left(E_{0}\right)$ :

$$
\begin{gathered}
J^{\prime}\left(E_{0}\right)= \\
\left(\begin{array}{cccccc}
-\left(\beta_{H}+\sigma_{H}\right) & 0 & 0 & b_{1} \alpha_{M H} S_{H}^{0} & 0 & b_{2} \alpha_{M w H} S_{H}^{0} \\
\beta_{H} & -\left(\gamma+\sigma_{H}+\sigma_{H}^{\prime}\right) & 0 & 0 & 0 & 0 \\
0 & b_{1} \alpha_{H M} S_{M}^{0} & -\left(\beta_{M}+\sigma_{M}\right) & 0 & 0 & 0 \\
0 & 0 & \beta_{M} & -\sigma_{M} & 0 & 0 \\
0 & b_{2} \alpha_{H M w} S_{M w}^{0} & 0 & 0 & -\left(\beta_{M w}+\sigma_{M}\right) & 0 \\
0 & 0 & 0 & 0 & \beta_{M w} & -\sigma_{M}
\end{array}\right) .
\end{gathered}
$$

It is easy to transform the submatrix $J^{\prime}\left(E_{0}\right)$ to a lower triangular matrix to obtain

$$
\begin{aligned}
& J^{\prime \prime}\left(E_{0}\right)= \\
& \left(\begin{array}{cccccc}
\left(\beta_{H}+\sigma_{H}\right)\left(\mathcal{R}_{C}^{2}-1\right) & 0 & 0 & 0 & 0 & 0 \\
\beta_{H} & -\left(\gamma+\sigma_{H}+\sigma_{H}^{\prime}\right) & 0 & 0 & 0 & 0 \\
0 & b_{1} \alpha_{H M} S_{M}^{0} & -\left(\beta_{M}+\sigma_{M}\right) & 0 & 0 & 0 \\
0 & 0 & \beta_{M} & -\sigma_{M} & 0 & 0 \\
0 & b_{2} \alpha_{H}{ }_{M} S_{M w}^{0} & 0 & 0 & -\left(\beta_{M w}+\sigma_{M}\right) & 0 \\
0 & 0 & 0 & 0 & \beta_{M w} & -\sigma_{M}
\end{array}\right) .
\end{aligned}
$$

All the eigenvalues of $J^{\prime \prime}\left(E_{0}\right)$ are negative or have negative real part if $\mathcal{R}_{C}^{2}<1$, or equivalently when $\mathcal{R}_{C}<1$, where

$$
\begin{aligned}
\mathcal{R}_{C}^{2}= & \frac{\beta_{H} b_{2} \beta_{M w} \alpha_{M w H} b_{2} \alpha_{H M w} S_{H}^{0} S_{M w}^{0}}{\sigma_{M}\left(\beta_{H}+\sigma_{H}\right)\left(\beta_{M w}+\sigma_{M}\right)\left(\gamma+\sigma_{H}+\sigma_{H}^{\prime}\right)} \\
& +\frac{\beta_{H} b_{1} \beta_{M} \alpha_{M H} b_{1} \alpha_{H M} S_{H}^{0} S_{M}^{0}}{\sigma_{M}\left(\beta_{H}+\sigma_{H}\right)\left(\beta_{M}+\sigma_{M}\right)\left(\gamma+\sigma_{H}+\sigma_{H}^{\prime}\right)} .
\end{aligned}
$$


Therefore, the disease-free equilibrium $E_{0}$ is locally asymptotically stable if $\mathcal{R}_{C}<1$. This implies that zika virus disease can be eliminated from the zika-endemic area, when $\mathcal{R}_{C}<1$, by using this control measure, if the initial sizes of the compartments are within the basin of attraction of the disease-free equilibrium.

\subsection{Existence of endemic equilibrium}

Let $E_{1}=\left(S_{H}^{*}, E_{H}^{*}, I_{H}^{*}, R_{H}^{*}, S_{M}^{*}, E_{M}^{*}, I_{M}^{*}, S_{M w}^{*}, E_{M w}^{*}, I_{M w}^{*}\right)$ where

$S_{H}^{*}=\frac{\Pi_{H}}{b_{1} \alpha_{M H} I_{M}^{*}+b_{2} \alpha_{M w H} I_{M}^{*}+\sigma_{H}}, E_{H}^{*}=\frac{b_{1} \alpha_{M H} I_{M}^{*}+b_{2} \alpha_{M w H} I_{M}^{*}}{\beta_{H}+\sigma_{H}}, I_{H}^{*}=\frac{\beta_{H} E_{H}^{*}}{\gamma+\sigma_{H}+\sigma_{H}^{\prime}}$, $R_{H}^{*}=\frac{\gamma I_{H}^{*}}{\sigma_{H}}, S_{M}^{*}=\frac{\Pi_{M}+q k \mu_{M} N_{M}^{*}}{b_{1} \alpha_{H M} I_{H}^{*}+\sigma_{M}}, E_{M}^{*}=\frac{b_{1} \alpha_{H M} S_{M}^{*} I_{H}^{*}}{\left(\beta_{M}+\sigma_{M}\right)}$,

$I_{M}^{*}=\frac{b_{1} \beta_{M} \alpha_{H M}\left(\Pi_{M}+q k \mu_{M} N_{M}^{*}\right) I_{H}^{*}}{\sigma_{M}\left(\beta_{M}+\sigma_{M}\right)\left(b_{1} \alpha_{H} I_{H}^{*}+\sigma_{M}\right)}, \quad S_{M w}^{*}=\frac{\Pi_{M w}+q k \mu_{M w} N_{M w}^{*}}{b_{1} \alpha_{H} M w I_{H}^{*}+\sigma_{M}}$,

$E_{M w}^{*}=\frac{b_{2} \alpha_{H M w} S_{M w}^{*} I_{H}^{*}}{\left(\beta_{M w}+\sigma_{M}\right)}, I_{M w}^{*}=\frac{b_{2} \beta_{M w} \alpha_{H M w}\left(\Pi_{M w}+k \mu_{M w} N_{M w}^{*}\right) I_{H}^{*}}{\sigma_{M}\left(\beta_{M w}+\sigma_{M}\right)\left(b_{2} \alpha_{H M w} I_{H}^{*}+\sigma_{M}\right)}$,

$N_{M}^{*}=\frac{\Pi_{M}}{\sigma_{M}-q k \mu_{M}}, N_{M w}^{*}=\frac{\Pi_{M w}}{\sigma_{M}-k \mu_{M w}}, N_{H}^{*}=\frac{\Pi_{H}-\sigma_{H}^{\prime} I_{H}^{*}}{\sigma_{H}}$.

This means that $E_{1}$ satisfies the following set of equations:

$$
\begin{aligned}
\Pi_{H}-b_{1} \alpha_{M H} S_{H} I_{M}-b_{2} \alpha_{M w H} I_{M w} S_{H}-\sigma_{H} S_{H} & =0, \\
b_{1} \alpha_{M H} I_{M} S_{H}+b_{2} \alpha_{M w H} I_{M w} S_{H}-\left(\beta_{H}+\sigma_{H}\right) E_{H} & =0, \\
\beta_{H} E_{H}-\left(\gamma+\sigma_{H}+\sigma_{H}^{\prime}\right) I_{H} & =0, \\
\gamma I_{H}-\sigma_{H} R_{H} & =0, \\
\Pi_{M}+q \kappa \mu_{M} N_{M}-b_{1} \alpha_{H} S_{M}(t) I_{H}-\sigma_{M} S_{M}(t) & =0, \\
b_{1} \alpha_{H M} S_{M}(t) I_{H}-\left(\beta_{M}+\sigma_{M}\right) E_{M}(t) & =0, \\
\beta_{M} E_{M}(t)-\sigma_{M} I_{M}(t) & =0, \\
\Pi_{M w}+\kappa \mu_{M w} N_{M w}-b_{2} \alpha_{H M w} S_{M w}(t) I_{H}-\sigma_{M} S_{M w}(t) & =0, \\
b_{2} \alpha_{H M w} S_{M w}(t) I_{H}-\left(\beta_{M w}+\sigma_{M}\right) E_{M w}(t) & =0, \\
\beta_{M w} E_{M w}(t)-\sigma_{M} I_{M w}(t) & =0,
\end{aligned}
$$

Let $I_{M}^{*}$ and $I_{M w}^{*}$ be written in the forms $I_{M}^{*}=\frac{A_{1} I_{H}^{*}}{A_{2} I_{H}^{*}+A_{3}}$ and $I_{M w}^{*}=$ $\frac{B_{1} I_{H}^{*}}{B_{2} I_{H}^{*}+B_{3}}$, respectively, where, $A_{1}=b_{1} \beta_{M} \alpha_{H M}\left(\Pi_{M}+q k \mu_{M} N_{M}^{*}\right), A_{2}=$ $\sigma_{M}\left(\beta_{M}+\sigma_{M}\right) b_{1} \alpha_{H M}, A_{3}=\sigma_{M}^{2}\left(\beta_{M}+\sigma_{M}\right) B_{1}=b_{2} \beta_{M w} \alpha_{H M w}\left(\Pi_{M w}+\right.$ $\left.k \mu_{M w} N_{M w}^{*}\right), B_{2}=\sigma_{M}\left(\beta_{M w}+\sigma_{M}\right) b_{2} \alpha_{H M w}, B_{3}=\sigma_{M}^{2}\left(\beta_{M w}+\sigma_{M}\right)$

Substituting these into (11) and simplifying, we obtain

$$
\frac{\beta_{H} \Pi_{H}}{\beta_{H}+\sigma_{H}} \frac{G_{1} I_{H}^{* 2}+G_{2} I_{H}^{*}}{G_{3} I_{H}^{* 2}+G_{4} I_{H}^{*}+G_{5}}-\left(\gamma+\sigma_{H}+\sigma_{H}^{\prime}\right) I_{H}^{*}=0
$$


where,

$G_{1}=b_{1} \alpha_{M H} A_{1} B_{2}+b_{2} \alpha_{M w H} B_{1} A_{2}, G_{2}=b_{1} \alpha_{M H} A_{1} B_{3}+b_{2} \alpha_{M w H} B_{1} A_{3}$, $G_{3}=G_{1}+\sigma_{H} A_{2} B_{2}, G_{4}=G_{2}+\sigma_{H}\left(A_{2} B_{3}+A_{3} B_{2}\right), G_{5}=\sigma_{H} A_{3} B_{3}$

Rearranging (19) gives the cubic equation

$$
-C_{1} I_{H}^{* 3}+C_{2} I_{H}^{* 2}+C_{3} I_{H}^{*}=0
$$

where $C_{1}=\left(\beta_{H}+\sigma_{H}\right)\left(\gamma+\sigma_{H}+\sigma_{H}^{\prime}\right) G_{3}, C_{2}=\left(\beta_{H} \Pi_{H} G_{1}-\left(\beta_{H}+\sigma_{H}\right)(\gamma+\right.$ $\left.\left.\sigma_{H}+\sigma_{H}^{\prime}\right)\right) G_{4}, C_{3}=\left(\beta_{H}+\sigma_{H}\right)\left(\gamma+\sigma_{H}+\sigma_{H}^{\prime}\right)\left[\mathcal{R}_{C}^{2}-1\right]$. The trivial solution, $I_{H}^{*}=0$, of (20) corresponds to the disease-free equilibrium, which is locally asymptotically stable if $\mathcal{R}_{C}<1$. Applying the Descartes rule of signs, we have

- case I: If $C_{2}<0$ and $\mathcal{R}_{C}>1$, a unique endemic equilibrium exists in the model.

- case II: If $C_{2}>0$ and $\mathcal{R}_{C}>1$, a unique endemic equilibrium exists in the model.

- case III: If $C_{2}<0$ and $\mathcal{R}_{C}<1$, no endemic equilibrium exists in the model.

- case IV: If $C_{2}>0$ and $\mathcal{R}_{C}<1$, two positive endemic equilibria exist in the model.

The first two cases indicate the absence of backward bifurcation in the model, since backward bifurcation involves at least two endemic equilibria. Case III, which is rare, indicates that only disease-free equilibrium exists in the model; it is an indication that the disease has been totally eradicated in the population. Case IV may be an indication of backward bifurcation in the model.

\subsection{Global asymptotic stability of the disease-free equilibrium}

We investigate the global stability of the disease-free equilibrium by using the two conditions (H1) and (H2) from Castillo-Chavez et al. (2012), which must be satisfied for $\mathcal{R}_{C}<1$. To do this, we rewrite the model equations in the form

$$
\begin{aligned}
& \frac{d X}{d t}=F_{1}(X, Y) \\
& \frac{d Y}{d t}=F_{2}(X, Y), F_{2}(X, 0) \geqslant 0,
\end{aligned}
$$

where $X=\left(S_{H}, R_{H}, S_{M}, S_{M w}\right)^{T}$ and $Y=\left(E_{H}, I_{H}, E_{M}, I_{M}, E_{M w}, I_{M w}\right)^{T}$ denote the uninfected and infected populations, respectively. The first condition (H1) requires that for $\frac{d X}{d t}=F_{1}(X, 0)$, the disease-free equilibrium 
$E_{0}=\left(\frac{\Pi_{H}}{\sigma_{H}}, 0, \frac{\Pi_{M}}{\sigma_{M}-q k \mu_{M}}, \frac{\Pi_{M w}}{\sigma_{M}-k \mu_{M w}}\right)$ must be globally asymptotically stable. The second condition (H2) requires that $\bar{F}_{2}(X, Y):=A Y-F_{2}(X, Y) \geqslant 0$, where $A$ is the Jacobian matrix of $F_{2}(X, Y)$ evaluated at the disease-free equilibrium. Using the first condition (H1), we solve

$$
\frac{d X}{d t}=F_{1}(X, 0)=\left(\begin{array}{c}
\Pi_{H}-\sigma_{H} S_{H} \\
-\sigma_{H} R_{H} \\
\Pi_{M}+q k \mu_{M} S_{M}-\sigma_{M} S_{M} \\
\Pi_{M w}+k \mu_{M w} S_{M w}-\sigma_{M} S_{M w}
\end{array}\right)
$$

The solution to $(23)$ is

$$
\begin{aligned}
S_{H}(t) & =\frac{\Pi_{H}}{\sigma_{H}}\left(1-e^{-\sigma_{H} t}\right)+S_{H}(0) e^{-\sigma_{H} t} \\
R_{H}(t) & =R_{H}(0) e^{-\sigma_{H} t} \\
S_{M}(t) & =\frac{\Pi_{M}}{\sigma_{M}-q k \mu_{M}}\left(1-e^{\left(\sigma_{M}-q k \mu_{M}\right) t}\right)+S_{M}(0) e^{\left(\sigma_{M}-q k \mu_{M}\right) t} \\
S_{M w}(t) & =\frac{\Pi_{M w}}{\sigma_{M}-q k \mu_{M w}}\left(1-e^{\left(\sigma_{M}-k \mu_{M w}\right) t}\right)+S_{M w}(0) e^{\left(\sigma_{M}-k \mu_{M w}\right) t} .
\end{aligned}
$$

Therefore, as

$t \rightarrow \infty,\left(S_{H}(t), R_{H}(t), S_{M}(t), S_{M w}(t)\right) \rightarrow\left(\frac{\Pi_{H}}{\sigma_{H}}, 0, \frac{\Pi_{M}}{\sigma_{M}-q k \mu_{M}}, \frac{\Pi_{M w}}{\sigma_{M}-k \mu_{M w}}\right)$ irrespective of the size of the initial populations.

Therefore, $E_{0}=\left(\frac{\Pi_{H}}{\sigma_{H}}, 0, \frac{\Pi_{M}}{\sigma_{M}-q k \mu_{M}}, \frac{\Pi_{M w}}{\sigma_{M}-k \mu_{M w}}\right)$ is globally asymptotically stable. For the second condition, we have that

$$
A=\left(\begin{array}{cccccc}
-\left(\beta_{H}+\sigma_{H}\right) & 0 & 0 & b_{1} \alpha_{M H} S_{H}^{0} & 0 & b_{2} \alpha_{M w H} S_{H}^{0} \\
\beta_{H} & -\left(\gamma+\sigma_{H}+\sigma_{H}^{\prime}\right) & 0 & 0 & 0 & 0 \\
0 & b_{1} \alpha_{H M} S_{M}^{0} & -\left(\beta_{M}+\sigma_{M}\right) & 0 & 0 & 0 \\
0 & 0 & \beta_{M} & -\sigma_{M} & 0 & 0 \\
0 & b_{2} \alpha_{H M w} S_{M w}^{0} & 0 & 0 & -\left(\beta_{M w}+\sigma_{M}\right) & 0 \\
0 & 0 & 0 & 0 & \beta_{M w} & -\sigma_{M}
\end{array}\right)
$$

and

$$
F_{2}(X, Y)=\left(\begin{array}{c}
b_{1} \alpha_{M H} I_{M} S_{H}+b_{2} \alpha_{M w H} I_{M w} S_{H}-\left(\beta_{H}+\sigma_{H}\right) E_{H}(t) \\
\beta_{H} E_{H}-\left(\gamma+\sigma_{H}+\sigma_{H}^{\prime}\right) I_{H}(t) \\
b_{1} \alpha_{H M} S_{M}(t) I_{H}-\left(\beta_{M}+\sigma_{M}\right) E_{M}(t) \\
\beta_{M} E_{M}(t)-\sigma_{M} I_{M}(t) \\
b_{2} \alpha_{H M w} S_{M w}(t) I_{H}-\left(\beta_{M w}+\sigma_{M}\right) E_{M w}(t) \\
\beta_{M w} E_{M w}(t)-\sigma_{M} I_{M w}(t)
\end{array}\right) .
$$


Then,

$$
\bar{F}_{2}(X, Y)=\left(\begin{array}{c}
\left(b_{1} \alpha_{M H} I_{M}+b_{2} \alpha_{M w H} I_{M w}\right)\left(S_{H}^{0}-S_{H}(t)\right) \\
0 \\
b_{1} \alpha_{H M}(t) I_{H}\left(S_{M}^{0}-S_{M}(t)\right) \\
0 \\
b_{2} \alpha_{H M w}(t) I_{H}\left(S_{M w}^{0}-S_{M w}(t)\right) \\
0
\end{array}\right) .
$$

This shows that $\bar{F}_{2}(X, Y) \geqslant 0$, since from $(24)$, we have that $S_{H}(t) \leqslant$ $S_{H}^{0}, S_{M}(t) \leqslant S_{M}^{0}, S_{M w}(t) \leqslant S_{M w}^{0}$. Hence, the disease-free equilibrium, $E_{0}$, is globally asymptotically stable. This result confirms the existence of a unique endemic equilibrium when $\mathcal{R}_{C}>1$. Therefore, backward bifurcation does not occur in the model.

\section{Numerical simulations}

Table 1. Parameter values used in this model

\begin{tabular}{cccccc}
\hline Parameter & Value & Source & Parameter & Value & Source \\
\hline$\Pi_{H}$ & 250 & assumed & $\sigma_{H}^{\prime}$ & 0.00001 & assumed \\
$\Pi_{M}$ & 500 & assumed & $\sigma_{H}$ & 0.0005 & assumed \\
$\alpha_{H M}$ & 0.175 & Gao et al. & $\Pi_{M w}$ & 250 & assumed \\
$\alpha_{H M w}$ & 0.0005 & assumed & $\mu_{M}$ & 0.03 & assumed \\
$\mu_{M w}$ & 0.03 & assumed & $\sigma_{M}$ & 0.15 & Ndii et al. \\
$\beta_{H}$ & $\frac{1}{3}$ & Kucharski et al. & $\kappa$ & 0.5 & Ndii et al. \\
$b_{1}$ & 0.0075 & assumed & $\gamma$ & 0.5 & assumed \\
$b_{2}$ & 0.7 & assumed & $\alpha_{M w H}$ & 0.001 & assumed \\
$\beta_{M}$ & $1 / 9$ & Dantas et al. & $\alpha_{M H}$ & 0.0175 & assumed \\
$\beta_{M w}$ & $1 / 18$ & assumed & $q$ & 0.017 & assumed \\
\hline
\end{tabular}

Numerical simulations are performed in this section to visualize the dynamics of zika virus disease in the presence of wolbachia-carrier mosquitoes used for disease control. The parameter values used in the simulations are given in Table 1. The initial solutions used are $S_{H}^{0}=1500, E_{H}^{0}=$ $200, I_{H}^{0}=500, R_{H}^{0}=100, S_{M}^{0}=1500, E_{M}^{0}=300, I_{M}^{0}=400, S_{M w}^{0}=$ $2400, E_{M w}^{0}=0, I_{M w}=0$. The results of the numerical simulation are presented graphically in Figures 1-4. In Figure 2, we see that the wolbachiacarrier mosquitoes suppress and replace the wild wolbachia-free mosquitoes in the wild. This replacement is achieved through cytoplasmic incompatibility, which prevents eggs of wolbachia-free mosquitoes from hatching. The effect of cytoplasmic incompatibility is visible in Figure 3, where the size 


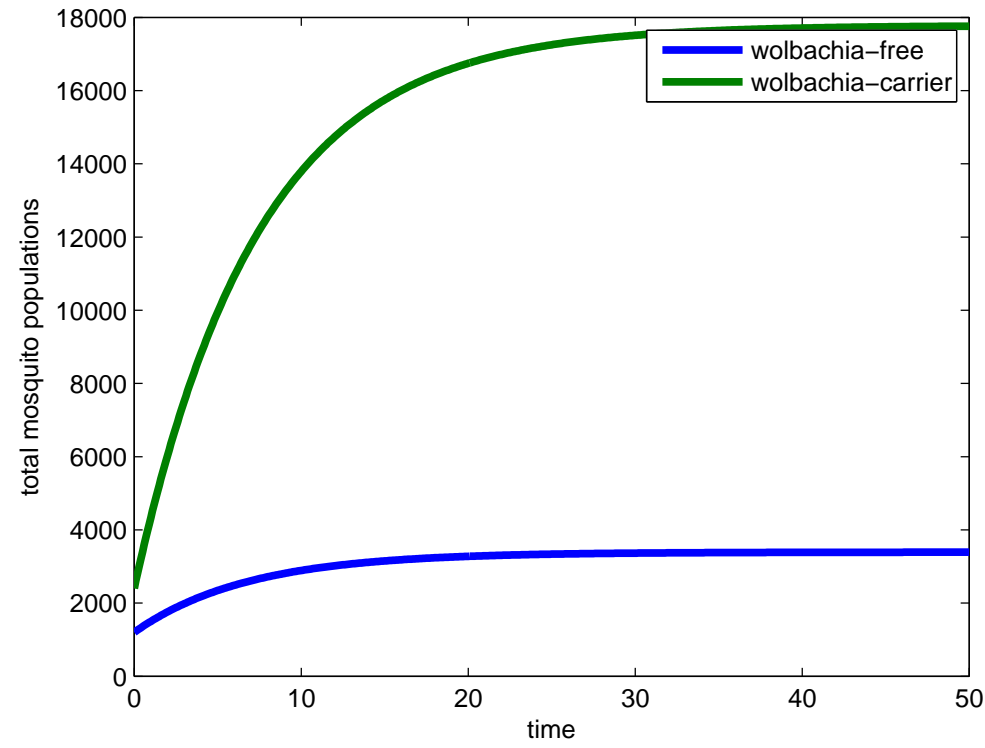

Figure 1. Total population of mosquitoes

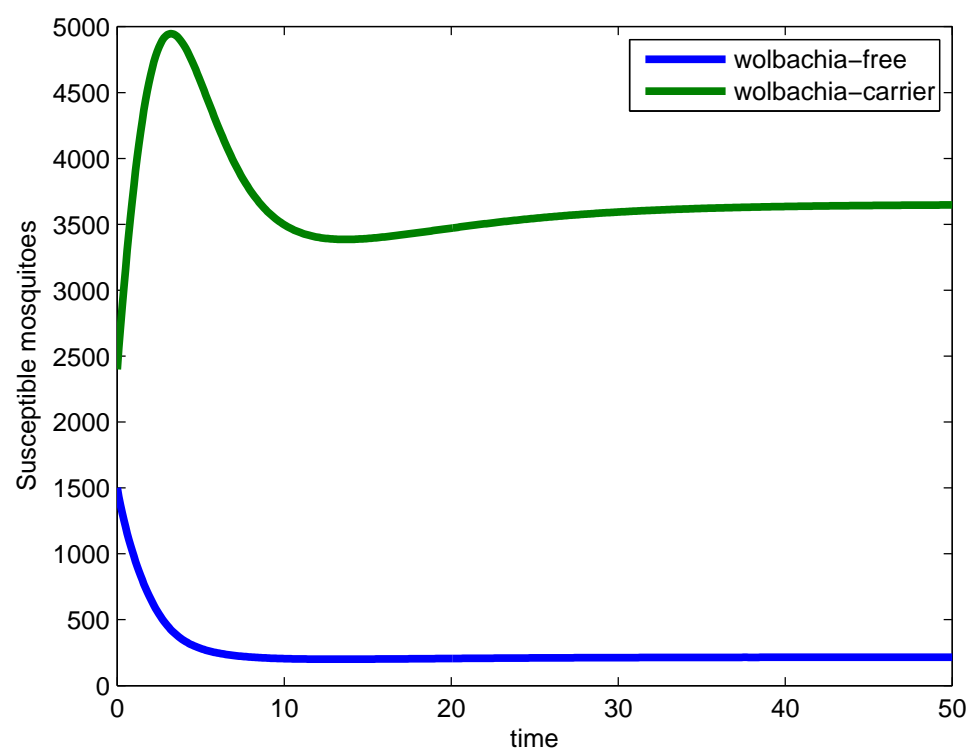

Figure 2. Mosquitoes susceptible to zika virus 


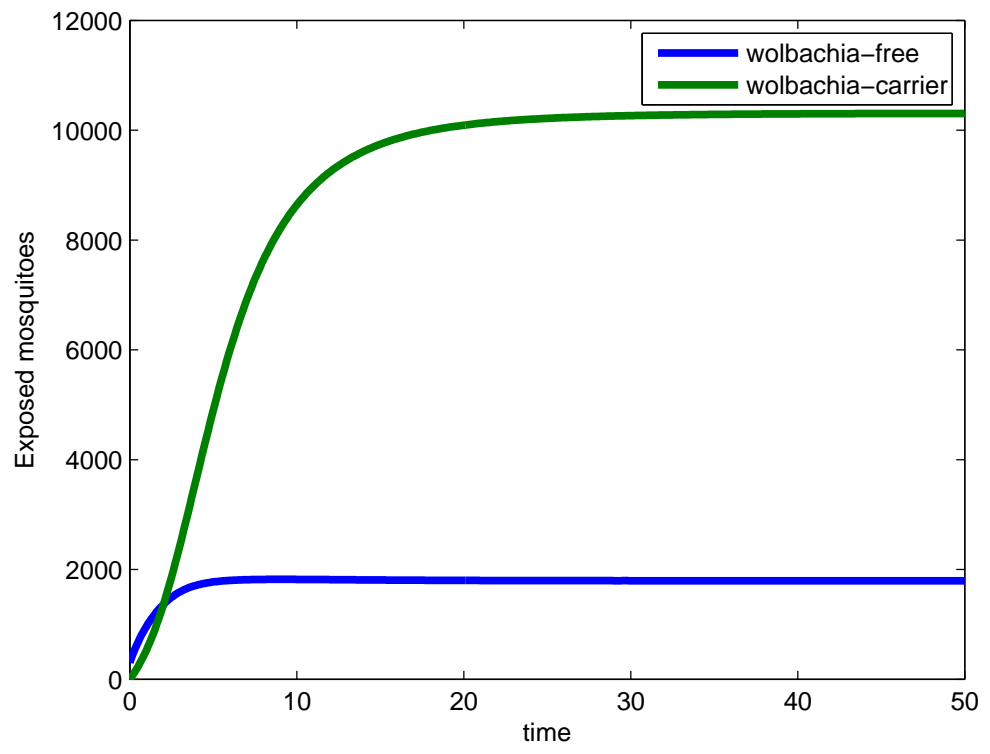

Figure 3. Mosquitoes exposed to zika virus

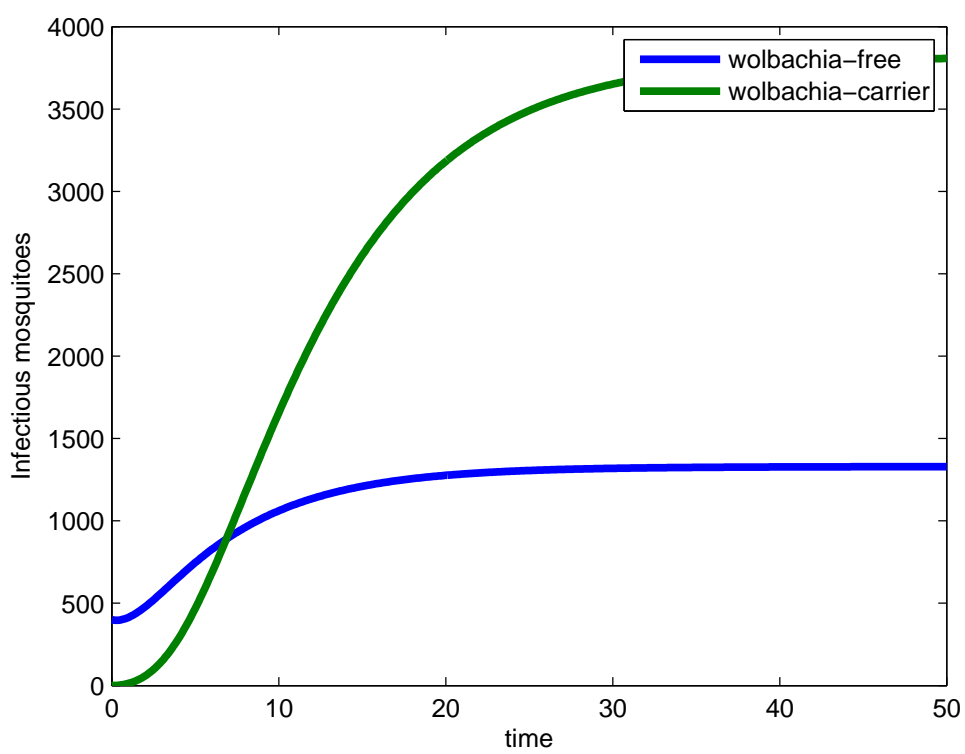

Figure 4. Mosquitoes that are infectious 
of the population of wolbachia-free mosquitoes that are susceptible to the virus is prevented from increasing. The same feature is seen in Figure 3 and Figure 4, where the wolbachia-free mosquitoes that are exposed to the zika virus and those that are infectious are seen to diminish over time, thereby reducing the rate at which zika virus spreads in the population.

\section{Conclusion}

In this paper, we have modeled the use of wolbachia-carrier Aedes aegypti mosquitoes to stop the spread of zika virus disease. We showed that the disease-free equilibrium of the model is both locally and globally asymptotically stable when the control reproduction number $\mathcal{R}_{C}<1$. Essentially, the global stability of the disease-free equilibrium entails that any sudden outbreak of zika virus disease will eventually subside over time, even when the initial sizes of the infected human and mosquito populations are high. Therefore, we conclude that this bio-control method is an effective and efficient method for controlling the spread of zika virus disease and other mosquito-borne diseases such as dengue, yellow fever and malaria.

\section{REFERENCES}

Cao-Lormeau V.M., Roche C., Teissier A., Robin E., Bery A.L., Mallet H.P., Sall A.A., Musso D. (2014): Zika Virus, French Polynesia, South Pacific, 2013. Emerging Infectious Diseases 20(6): 1085-1086.

Cao-Lormeau V.M., Blake A., Mons S., Lastere S., Roche C., Vanhomwegen J., Dub T., Baudouin L., Teissier A., Larre P., Vial A. L., Decam C., Choumet V., Halstead S.K., Neil J., ..., Ghawché F. (2016): Guillain-Barre Syndrome outbreak associated with Zika virus infection in French Polynesia: a casecontrol study. Lancet 387(10027): 1531-1539.

Castillo-Chavev C., Feng Z., Huang W. (2012): On the Computation of $R_{0}$ and its role on global stability. Mathematical Approaches for Emerging and Reemerging Infectious Diseases 1 (2012): 229.

Cauchemez S., Besnard M., Bompard P., Dub T., Guillemette-Artur P., EyrolleGuignot D., Salje H., ..., Mallet, H. (2016): Association between Zika virus and microcephaly in French Polynesia, 2013-15: a retrospective study. Lancet $387: 2125$.

Dantas E., Tosin M., Cunha A. Jr. (2017): Calibration of a SEIR epidemic model to describe Zika virus outbreak in Brazil. $<$ hal-01456776v2 $>$

Diekmann O., Heesterbeek J.A.P., Roberts M.G. (2010): The construction of nextgeneration matrices for compartmental epidemic models. Journal of Royal Society Interface 7: 873-885. 
Gao D., Lou Y., He D., Porco T.C., Kuang Y., Chowell G., Ruan S. (2016): Prevention and Control of Zika as a Mosquito-Borne and Sexually Transmitted Disease: A Mathematical Modeling Analysis. Scientific Reports 6: 28070.

Hancock P.A., Sinkins S.P., Godfray H.J. (2011): Population dynamic models of the Spread of Wolbachia. The American Naturalist 177(3): 323-333.

Hughes H., Britton N.F. (2013): Modelling the use of Wolbachia to control dengue fever transmission. Bulletin of Mathematical Biology 75 (5): 796-818.

Koiller J., Da Silva M., Souza M., Codeco C., Iggidr A., Salliet, G. (2014): Aedes, Wolbachia and Dengue. Inria, France, Research Report RR-8462.

Kucharski A.J., Funk S., Eggo R. M., Mallet H.P., Edmunds J., Nilles, E.J. (2016): Transmission dynamics of Zika virus in island populations: a modelling analysis of the 2013-14 French Polynesia outbreak. Neglected Tropical Diseases 10(5): bioRxiv. 2016;p. 038588.

Mains J.W., Brelsfoard C.L., Crain P.R., Huang Y., Dobson S.L. (2013): Population Impacts of Wolbachia on Aedes albopictus. Ecological Applications 23: 493-501.

Mlakar J., Korva M., Tul N., Popović, M., Avšič Ž.T. (2016): Zika Virus Associated with Microcephaly. New England Journal of Medicine, 374(10): 951-958.

Moreno V., Espinoza B., Bichara D., Holechek S.A., Castillo-Chavez C. (2016): Role of short-term dispersal on the dynamics of Zika virus. arXiv preprint arXiv: 1603.00442

Musso D., Nhan T., Robin E., Roche C., Bierlaire D., Zisou K., Shan Y. A., Cao-Lormeau, V.M., Broult J. (2014): Potential for Zika virus transmission through blood transfusion demonstrated during an outbreak in French Polynesia, November 2013 to February 2014. Euro Surveillance 19(14): 20761.

Ndii M.Z., Hickson R.I., Mercer G.N. (2002): Modelling the Introduction of Wolbachia into Aedes Aegypti Mosquitoes to Reduce Dengue Transmission. The ANZIAM Journal 53: 1-15.

World Health Organization (WHO), WHO statement on the first meeting of the International Health Regulations (2015) Emergency Committee on Zika virus and observed increase in neurological disorders and neonatal malformations, February 1, 2016. http://www.who.int/mediacentre/news/statements/2016/1st-emergencycommittee-zika/en/ 